\title{
Qualitative Properties of Autocatalytic Reactions Occurring in a Flow System
}

\author{
Sang H. Kim and Vladimir Hlavacek \\ Department of Che mical Engineering, State University of New York at Buffalo, \\ Buffalo, New York 14260, USA
}

Z. Naturforsch. 40 a, 736-747 (1985); received March 29, 1985

The dynamic behavior of an autocatalytic reaction with a product inhibition term is studied in a flow system. A unique steady state exists in the continuous tank reactor. Linear stability analysis predicts either a stable node, a focus or an unstable saddle-focus. Sustained oscillations around the unstable focus can occur for high values of the Damköhler number (Da). In the distributed system, travelling, standing or complex oscillatory waves are detected. For a low value of $\mathrm{Da}$, travelling waves with a pseudo-constant pattern are observed. With an intermediate value of $\mathrm{Da}$, single or multiple standing waves are obtained. The temporal behavior indicates also the appearance of retriggering or echo waves. For a high value of $\mathrm{Da}$, both single peak and complex multipeak oscillations are found. In the cell model, both regular oscillations near the inlet and chaotic behavior downstream are observed. In the dispersion model, higher Peclet numbers $(\mathrm{Pe})$ eliminate the oscillations. The spatial profile shows a train of pulsating waves for the discrete model and a single pulsating or solitary wave for the continuous model.

\section{Introduction}

Autocatalytic reactions represent a very important class of chemical reactions with a variety of applications in combustion, biological reactors, enzyme systems, and homogeneous and catalytic reactors. To mention only a few: Lotka's model on population dynamics [1], the Brusselator description of a theoretical tri-molecular reaction [2-5], the Belousov-Zhabotinskii reaction $[6,7]$, the modified Lotka-Volterra model for hydrocarbon oxidation and cool flames [8], and Yamazaki's reaction [9].

There are striking parallels between isothermal autocatalytic and exothermic first-order reactions. While there is a systematic analysis available on exothermic reaction systems, no extensive study has been made of the corresponding isothermal autocatalytic prototypes where the feedback is not thermal but autocatalytic. Fascinating dissipative structures (spatially, temporally or even spatiotemporally organized states) have been studied both theoretically and experimentally [2-7]. Among them, symmetry breaking structures, wave trains including planar and standing waves, and target,

Reprint requests to Prof. V. Hlavacek, Department of Chemical Engineering, State University of New York at Buffalo, Buffalo, New York 14260, USA. spiral and scroll patterns as well as chaotic behavior have drawn the attention of mathematicians, physicists, biologists, chemists and chemical engineers.

The Belousov-Zhabotinskii reaction, oxidation of malonic acid by potassium bromate with ceric/ cerous ions as a catalyst, has been extensively studied. Tatterson and Hudson [10] observed chemical waves of pulse type propagating in a tube, in the absence of convection effects. Frequency, speed and wavelength did not change significantly during the process. This observation reveals the rich spectrum of wave phenomena in the BelousovZhabotinskii reaction [11]. Marek and Svobodová [12] observed expermentally sustained oscillations and jump phenomena (transition of oscillatory behavior to a steady state) in a continuous stirred tank reactor (CSTR) and standing as well as travelling waves in tubular flow reactors. Schmitz, Hudson and Graziani [13-14] established simple singlepeak and complex multipeak oscillations, and chaotic behavior in a CSTR. Rössler [15] also observed an irregular screw-type chaotic behavior in a CSTR. Simoyi et al. [16] noticed subharmonic bifurcations of a limit cycle leading to alternating complex periodic and chaotic regimes in a CSTR. Roux [17] studied experimentally bifurcations of a limit cycle leading to quasi-periodic oscillations. 
To our knowledge, no systematic studies have been reported on the dynamic of autocatalytic reactions occurring in distributed flow systems, for example packed bed tubular reactors. In this paper we attempt to comprehend the interplay of transport processes, such as diffusion, convection and complex autocatalytic kinetics. One-dimensional (1D) and two-dimensional (2D) models are considered. Emphasis is placed on the problems of local stability in a CSTR, wave propagation phenomena for standing, travelling and spatio-temporal waves, and chaotic behavior. Possible defferences between discrete and continuous descriptions of distributed parameter flow systems will be discussed.

\section{Governing Equations}

Consider the following chain of reactions containing an autocatalytic step in quadratic form. This model gives rise to undamped oscillations in a closed system [18]. The reaction exhibits a kind of backward product inhibition as shown in (1) and (2);

$$
\begin{aligned}
& \rightarrow C_{1} \underset{k_{1}}{\stackrel{\downarrow}{\rightarrow}} C_{2} \underset{k_{2}}{\rightarrow} C_{3} \underset{k_{3}}{\stackrel{\downarrow}{\rightarrow}} \text { products. } \\
& \frac{\mathrm{d} C_{1}}{\mathrm{~d} t}=k_{1} C_{1}-k_{3} C_{1} C_{3}, \\
& \frac{\mathrm{d} C_{2}}{\mathrm{~d} t}=k_{1} C_{1}-k_{2} C_{2}, \\
& \frac{\mathrm{d} C_{3}}{\mathrm{~d} t}=k_{2} C_{2}-k_{3} C_{3} .
\end{aligned}
$$

In this paper we are analyzing an isothermal autocatalytic system occurring in a packed bed. Such systems are typical for immobilized enzymes deposited on a solid matrix. We formally get the same description for an autocatalytic homogeneous reaction taking place in a tubular system packed with inert particles. Below we will make use of models developed for the simulation of packed bed reactors.

Two distinct types of description of mass dispersion in packed bed reactors have been adopted so far. The dispersions model assumes that the transport may be phrased in terms of continuous description while the cell model visualizes the behavior of packed bed reactors by a sequence of well-stirred tank reactors as shown in Fig. 1. It has been shown in the literature [19-20] that certain differences, particularly concerning multiplicity, do exist between the cell and dispersion models for an exothermic first-order reaction. Therefore, both the cell model with backflow and the dispersion model are considered. Isothermal conditions, an identical volume of each cell, the same back-flow rate for all components, and no changes of physical properties are assumed in the present paper.

\subsection{One-dimensional Systems}

The mass balance around the $i$-th tank in the cell model (cf. Fig. 1) yields the following dimensionless differential-difference equations:

$$
\begin{aligned}
\frac{\mathrm{d} U_{i}}{\mathrm{~d} \tau}= & \left(1+K_{m}\right) U_{i-1}-\left(1+2 K_{m}\right) U_{i}+K_{m} U_{i+1} \\
& +\mathrm{Da}\left(U_{i}-\alpha_{1} U_{i} W_{i}\right), \\
\frac{\mathrm{d} V_{i}}{\mathrm{~d} \tau}= & \left(1+K_{m}\right) V_{i-1}-\left(1+2 K_{m}\right) V_{i}+K_{m} V_{i+1} \\
& +\mathrm{Da}\left(U_{i}-\alpha_{2} V_{i}\right), \\
\frac{\mathrm{d} W_{i}}{\mathrm{~d} t}= & \left(1+K_{m}\right) W_{i-1}-\left(1+2 K_{m}\right) W_{i}+K_{m} W_{i+1} \\
& +\mathrm{Da}\left(\alpha_{2} V_{i}-\alpha_{3} W_{i}\right) .
\end{aligned}
$$

The balance of the two fictitious cells, 0 and $N+1$, gives

$$
\begin{array}{ll}
i=0: \quad\left(1+K_{m}\right) U_{0}=1+K_{m} U_{1}, \\
& \left(1+K_{m}\right) V_{0}=K_{m} V_{1}, \\
& \left(1+K_{m}\right) W_{0}=K_{m} W_{1} . \\
i=N+1: \quad & U_{N+1}=U_{N}, \quad V_{N+1}=V_{N}, \quad W_{N+1}=W_{N} .
\end{array}
$$

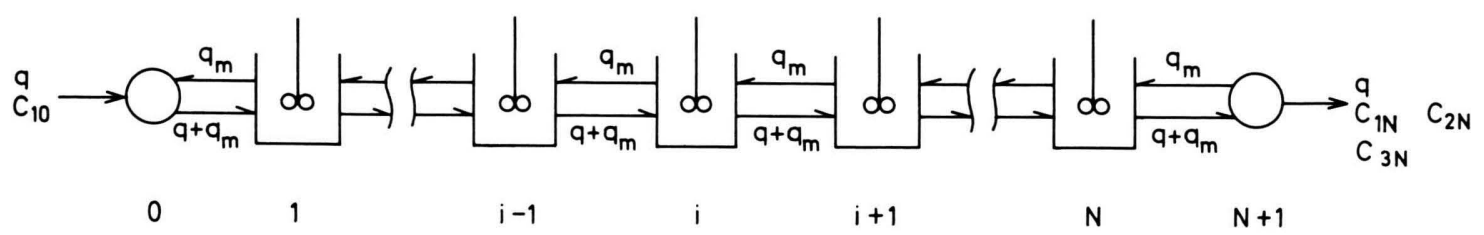

Fig. 1. Schematic sketch of the cell model with backflow, $q_{\mathrm{m}}$. 
The corresponding dispersion model is described by three coupled partial differential equations of parabolic type:

$$
\begin{aligned}
& \frac{\partial U}{\partial \tau}=\frac{1}{\mathrm{Pe}_{x}} \frac{\partial^{2} U}{\partial x^{2}}-\frac{\partial U}{\partial x}+\mathrm{Da}\left(U-\alpha_{1} U W\right), \\
& \frac{\partial V}{\partial \tau}=\frac{1}{\mathrm{Pe}_{x}} \frac{\partial^{2} V}{\partial x^{2}}-\frac{\partial V}{\partial x}+\mathrm{Da}\left(U-\alpha_{2} V\right), \\
& \frac{\partial W}{\partial \tau}=\frac{1}{\mathrm{Pe}_{x}} \frac{\partial^{2} W}{\partial x^{2}}-\frac{\partial W}{\partial x}+\mathrm{Da}\left(\alpha_{2} V-\alpha_{3} W\right),
\end{aligned}
$$

subject to Danckwerts boundary conditions

$$
\begin{gathered}
x=0, \tau>0: \quad 1=U-\frac{1}{\mathrm{Pe}_{x}} \frac{\partial U}{\partial x}, \\
V=\frac{1}{\mathrm{Pe}_{x}} \frac{\partial V}{\partial x}, \\
W=\frac{1}{\mathrm{Pe}_{x}} \frac{\partial W}{\partial x} . \\
x=N, \tau>0: \quad \frac{\partial U}{\partial x}=\frac{\partial V}{\partial x}=\frac{\partial W}{\partial x}=0 .
\end{gathered}
$$

Here we have denoted by $U, V, W$ the dimensionless concentrations of $C_{1}, C_{2}$ and $C_{3}$, respectively. $\alpha_{1}, \alpha_{2}, \alpha_{3}$ are the dimensionless kinetic constants, $\mathrm{Da}$ is the Damköhler number, $K_{m}$ the mass backflow ratio, $\mathrm{Pe}_{x}$ axial particle Peclet number for mass, and $\tau$ the dimensionless time, defined, respectively, by

$$
\begin{aligned}
U_{i}=\frac{C_{1, i}}{C_{10}}, & V_{i}=\frac{C_{2, i}}{C_{10}},
\end{aligned}
$$

\subsection{Two-dimensional Systems}

The mass balance for the Deans-Lapidus cell model [21], based on a 2-dimensional network of perfectly stirred tanks in a cylindrical packed bed reactor, yields a set of ordinary differential equations, see (8). Here $i$ and $j$ denote the cell number in the axial and radial directions, respectively. $K_{r}$ represents the exchange coefficient in the radial direction.

$$
\begin{aligned}
\frac{\mathrm{d} U_{i, j}}{\mathrm{~d} \tau}= & \left(\frac{j-\frac{3}{4}}{2 j-1}\right) U_{i-1, j-\frac{1}{2}}+\left(\frac{j-\frac{1}{4}}{2 j-1}\right) U_{i-1, j+\frac{1}{2}}-U_{i, j} \\
& +\left(\frac{K_{r}}{2 j-1}\right)\left(U_{i, j-1}-2 U_{i, j}+U_{i, j+1}\right) \\
& +\mathrm{Da}\left(U_{i, j}-\alpha_{1} U_{i, j} W_{i, j}\right), \\
\frac{\mathrm{d} V_{i, j}}{\mathrm{~d} \tau}= & \left(\frac{j-\frac{3}{4}}{2 j-1}\right) V_{i-1, j-\frac{1}{2}}+\left(\frac{j-\frac{1}{4}}{2 j-1}\right) V_{i-1, j+\frac{1}{2}}-V_{i, j} \\
& +\left(\frac{K_{r}}{2 j-1}\right)\left(V_{i, j-1}-2 V_{i, j}+V_{i, j+1}\right) \\
& +\mathrm{Da}\left(U_{i, j}-\alpha_{2} V_{i, j}\right), \\
\frac{\mathrm{d} W_{i, j}}{\mathrm{~d} \tau}= & \left(\frac{j-\frac{3}{4}}{2 j-1}\right) W_{i-1, j-\frac{1}{2}}+\left(\frac{j-\frac{1}{4}}{2 j-1}\right) W_{i-1, j+\frac{1}{2}}-W_{i, j} \\
& +\left(\frac{K_{r}}{2 j-1}\right)\left(W_{i, j-1}-2 W_{i, j}+W_{i, j+1}\right) \\
& +\mathrm{Da}\left(\alpha_{2} V_{i, j}-\alpha_{3} W_{i, j}\right),
\end{aligned}
$$

where

$$
\begin{array}{rlrl}
i & =1,2,3 \ldots, N, \\
j & =\frac{1}{2}, \frac{3}{2}, \frac{5}{2}, \ldots, \frac{2 M-1}{2} & \text { for } \quad i=\text { odd } \\
& =1,2,3, \ldots, M & \text { for } \quad i=\text { even }
\end{array}
$$

subject to the boundary conditions

$$
\begin{aligned}
i=1, j>0: & \mathrm{U}_{0, j \pm \frac{1}{2}}=1.0, \\
& V_{0, j \pm \frac{1}{2}}=0.0, \\
& W_{0, j \pm \frac{1}{2}}=0.0 .
\end{aligned}
$$

$$
\begin{array}{ll}
j=\frac{1}{2}, i=\text { odd }: & U_{i-1,0}=0.0, \\
& V_{i-1,0}=0.0, \\
& W_{i-1,0}=0.0 . \\
& U_{i, 0}=U_{i, 2}, \\
& V_{i, 0}=V_{i, 2}, \\
& W_{i, 0}=W_{i, 2} . \\
j=\frac{2 M-1}{2}, i=\text { odd }: & U_{i-1, M}=U_{i-1, M-1}, \\
& V_{i-1, M}=V_{i-1, M-1}, \\
& W_{i-1, M}=W_{i-1, M-1} .
\end{array}
$$




$$
j=M, i=\text { even } \begin{aligned}
U_{i, M+1} & =U_{i, M-1}, \\
V_{i, M+1} & =V_{i, M-1}, \\
W_{i, M+1} & =W_{i, M-1} .
\end{aligned}
$$

\section{Analysis and Numerical Results}

Numerical integration of the sets of ordinary differential equations (3)-(4) and (8)-(9) was performed using Gear's method for integration of stiff systems of ordinary differential equations. The error of integration was controlled to six decimal places. The set of nonlinear parabolic partial differential equations (5) - (6) was integrated by a CrankNicolson method with an automatic time-step adjustment. The error of integration was controlled to four significant decimal places. The majority of the calculations was performed using forty mixing cells in the axial and seven in the radial directions. In order to compare the results from both models, the dimensionless length, $x$, in the dispersion model was converted into an equivalent number of cells in the corresponding cell model.

\subsection{Lumped Parameter Systems}

A detailed understanding of lumped parameter systems may provide a deeper insight into the dynamics of the corresponding distributed systems. For a single CSTR, (3) is simplified to a threevariable system represented by (10):

$$
\begin{aligned}
& \frac{\mathrm{d} U}{\mathrm{~d} \tau}=1-U+\mathrm{Da}\left(U-\alpha_{1} U W\right)=f_{1}(U, V, W), \\
& \frac{\mathrm{d} V}{\mathrm{~d} \tau}=-V+\mathrm{Da}\left(U-\alpha_{2} V\right)=f_{2}(U, V, W), \\
& \frac{\mathrm{d} W}{\mathrm{~d} \tau}=-W+\mathrm{Da}\left(\alpha_{2} V-\alpha_{3} W\right)=f_{3}(U, V, W) .
\end{aligned}
$$

The presence of multiple steady states in (10) can be evaluated by setting $\frac{\mathrm{d} U}{\mathrm{~d} \tau}, \frac{\mathrm{d} V}{\mathrm{~d} \tau}$ and $\frac{\mathrm{d} W}{\mathrm{~d} \tau}$ equal to zero and analyzing the resulting set of three nonlinear algebraic equations. Among the four governing parameters in (10), the Damköhler number (Da) is the most important parameter for the understanding the properties of the flow system. After simple algebraic manipulations a quadratic equation results for the concentration $U$ (the subscript s refers to steady state conditions):

$$
a_{1} U_{\mathrm{s}}^{2}+a_{2} U_{\mathrm{s}}+a_{3}=0 .
$$

The constants $a_{1}, a_{2}$ and $a_{3}$ are defined by

$$
\begin{aligned}
& a_{1}=\alpha_{1} \alpha_{2} \mathrm{Da}^{3}, \\
& a_{2}=(1-\mathrm{Da})\left(1+\alpha_{2} \mathrm{Da}\right)\left(1+\alpha_{3} \mathrm{Da}\right), \\
& a_{3}=-\left(1+\alpha_{2} \mathrm{Da}\right)\left(1+\alpha_{3} \mathrm{Da}\right) .
\end{aligned}
$$

Although multiple steady states are frequently observed in autocatalytic reaction systems, our analysis revealed that for the autocatalytic system in question, multiple solutions do not exist.

The local stability properties in a region close to the steady state can be predicted from the eigenvalues, $\lambda$, satisfying the cubic equation

$$
\lambda^{3}+b_{1} \lambda^{2}+b_{2} \lambda+b_{3}=0 \text {. }
$$

Here the constants $b_{1}-b_{3}$ can be obtained as follows:

$$
\begin{gathered}
b_{1}=-\left\{\left(\frac{\partial f_{1}}{\partial U}\right)_{s}+\left(\frac{\partial f_{2}}{\partial V}\right)_{s}+\left(\frac{\partial f_{3}}{\partial W}\right)\right\}, \\
b_{2}=\left(\frac{\partial f_{1}}{\partial U}\right)_{\mathrm{s}}\left(\frac{\partial f_{2}}{\partial V}\right)_{\mathrm{s}}+\left(\frac{\partial f_{1}}{\partial U}\right)_{\mathrm{s}}\left(\frac{\partial f_{3}}{\partial W}\right)+\left(\frac{\partial f_{2}}{\partial V}\right)_{\mathrm{s}}\left(\frac{\partial f_{3}}{\partial W}\right)_{\mathrm{s}}, \\
b_{3}=-\left\{\left(\frac{\partial f_{1}}{\partial U}\right)_{\mathrm{s}}\left(\frac{\partial f_{2}}{\partial V}\right)_{\mathrm{s}}\left(\frac{\partial f_{3}}{\partial W}\right)_{\mathrm{s}}\right. \\
\left.+\left(\frac{\partial f_{1}}{\partial W}\right)_{\mathrm{s}}\left(\frac{\partial f_{2}}{\partial U}\right)_{\mathrm{s}}\left(\frac{\partial f_{3}}{\partial V}\right)_{\mathrm{s}}\right\} .
\end{gathered}
$$

Linear stability analysis shows that a stable node and a focus, and an unstable saddle-focus may exist in the CSTR as shown in Figure 3. A stable limit cycle may occur around the unstable steady state (see Figure 2). Hopf-bifurcation points occur under an increasing value of the kinetic constant $\alpha_{1}$ (cf. Figure 2). The oscillatory behavior exists in the region of higher Da's, i.e., at low flow rates with higher feed concentrations. Low flow rates favor the oscillations while higher flow rates result in the steady state mode of operation (see Figures 2-4). These trends are in accordance with experimental observations on the Belousov-Zhabotinskii reaction as taking place in a CSTR [13-16]. Isolas and mushrooms in the steady state diagrams "concentration versus flow rates", which Gray observed for 


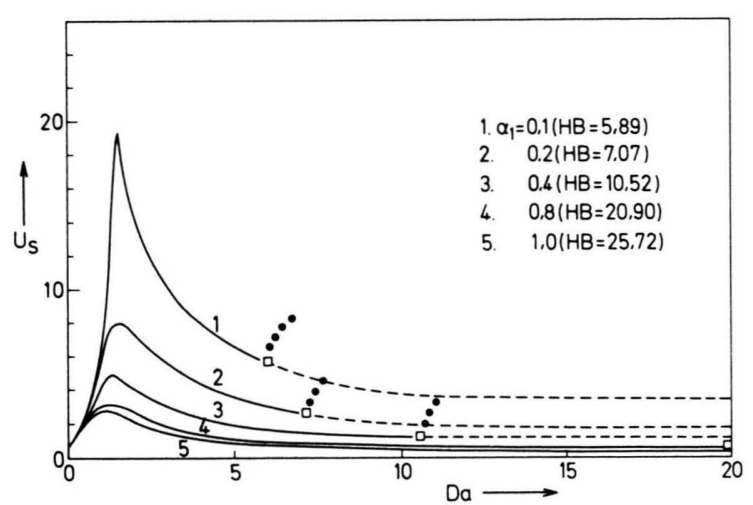

Fig. 2. Steady states versus Damköhler number in the CSTR. $\alpha_{2}=\alpha_{3}=0.2$ ( $\square$ and HB denote Hopf-bifurcation points). _ stable steady state; - - - unstable steady state; $\bullet \bullet \bullet$ stable periodic solution.

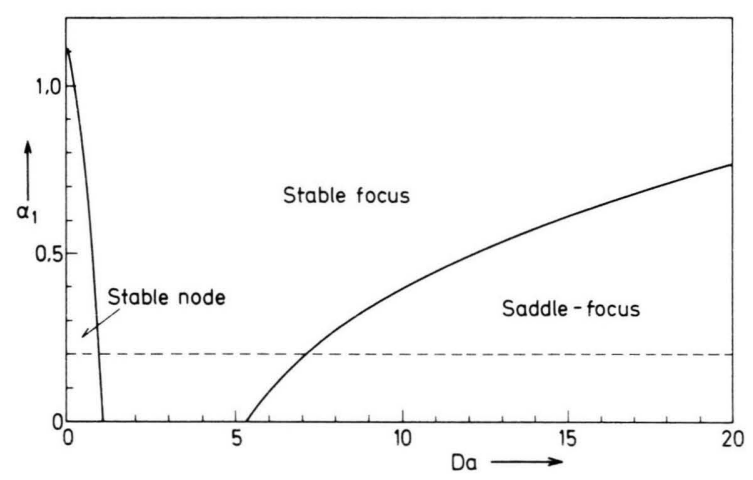

Fig. 3. Local stability region in the CSTR. $\alpha_{2}=\alpha_{3}=0.2$.

his quadratic and cubic autocatalytic reactions [22], are not detected in our system.

The period of the oscillations increases with decreasing values of $\mathrm{Da}$, see Figure 5. This result is in qualitative agreement with experimental observations obtained by Marek [12] on the BelousovZabotinskii reaction in a CSTR.

\subsection{Distributed Parameter Systems}

Wave phenomena in distributed systems can be qualitatively explained as a kind of interaction between two or more coupled or forced oscillators. Pismen [23] analyzed conditions for spatial and/or temporal order to emerge from a homogeneous steady state: such as inhomogeneous oscillating

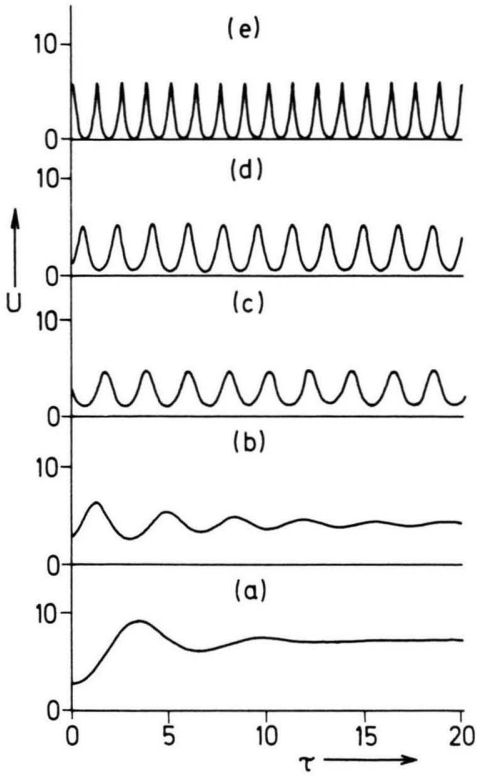

Fig. 4. Time evolution depending on the Damköhler number in the CSTR. $\alpha_{1}=\alpha_{2}=\alpha_{3}=0.2$. Da is: (a) 2.0, (b) 4.0, (c) 8.0 , (d) 10.0 , (e) 16.0 .

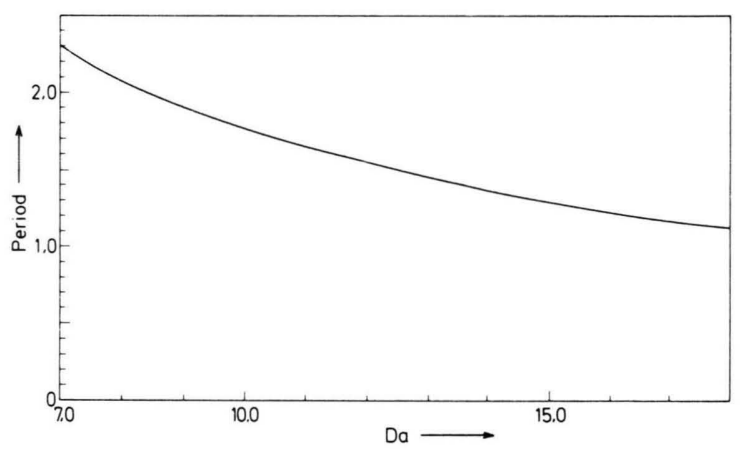

Fig. 5. Period of oscillations versus Damköhler number in the CSTR. $\alpha_{1}=\alpha_{2}=\alpha_{3}=0.2$.

states (wave patterns). Neu [24], using singular perturbation analysis around homogeneous oscillatory states, studied in detail the phase desynchronization of two coupled oscillators in order to develop criteria for wave propagation.

Apart from phase desynchronization, external gradients and inhomogeneities, the geometry of the system, and the initial conditions can also play an important role in wave propagation phenomena (target, spiral waves and scroll patterns). 


\section{(1) One-dimensional Systems}

The analysis presented below considers only one component, $C_{1}$, in the feed $\left(C_{2}=C_{3}=0\right)$. The initial conditions considered are $U=3.0, \quad V=W$ $=4.0$.

As shown in Figs. 6-11, travelling waves are observed at low Da's while standing waves prevail at intermediate values of $\mathrm{Da}$. The Damköhler number can be expressed as the ratio of the characteristic time for bulk mass flow to that for the chemical reaction. Therefore, high flow rates favor travelling wave phenomena while intermediate flow rates support the occurrence of standing waves.

The velocity of the travelling wave increases slightly during the initial stage, passes through a maximum, and then decreases again before approaching a particular stable structure in space. The shape of the travelling wave depicted in Figs. 6-7 is continually changing. The trend is typical for problems featuring high values of $\mathrm{Da}$.

The steady state profiles presented in Figs. 6-11 show the typical features of a spatial structure. An increasing value of $\mathrm{Da}$ modifies the wave pattern from a travelling to a standing wave. The transition occurs around $\mathrm{Da} \approx 0.1$. The standing waves in Figs. 10-11 remind one of the characteristics of a triggering wave emerging from a uniform homogeneous state. A single spatial structure (or standing wave) appears in Figs. 8-9 in the region near the inlet.

With increasing values of Da, Figs. $10-11$, two or more triggering waves or multiple standing waves appear in the system, after the first standing wave already has developed upstream near the inlet. This

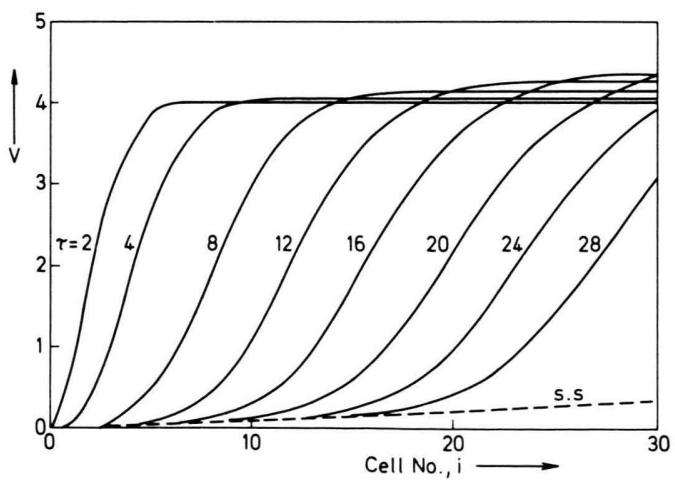

Fig. 6. Travelling waves in a tubular system. $\mathrm{Da}=0.01$, $\alpha_{1}=\alpha_{2}=\alpha_{3}=0.2$ (s.s. denotes steady states).

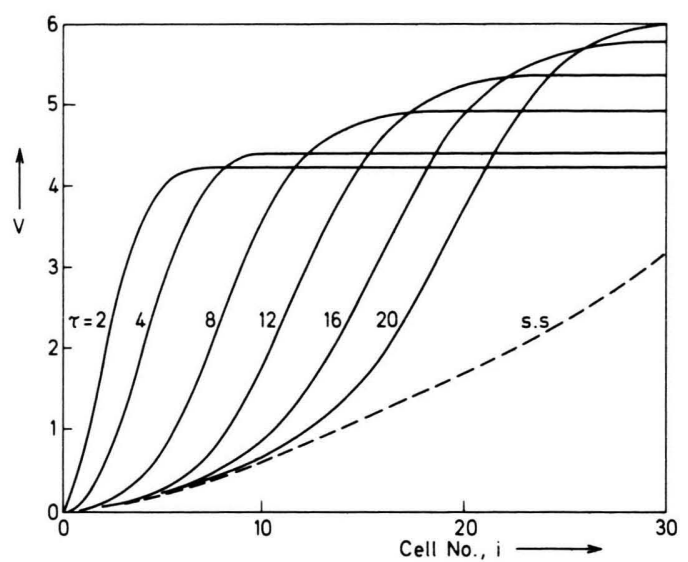

Fig. 7. Travelling waves in a tubular system. $\mathrm{Da}=0.05$, $\alpha_{1}=\alpha_{2}=\alpha_{3}=0.2$.

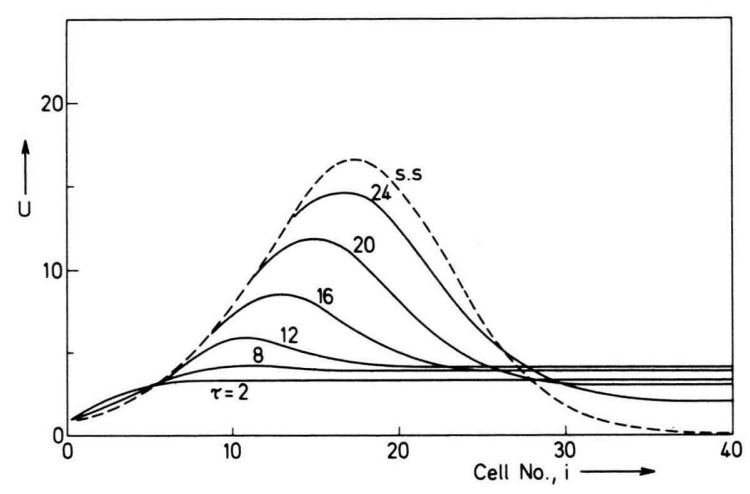

Fig. 8. Single standing wave in a tubular system. $\mathrm{Da}=0.20$, $\alpha_{1}=\alpha_{2}=\alpha_{3}=0.2$.

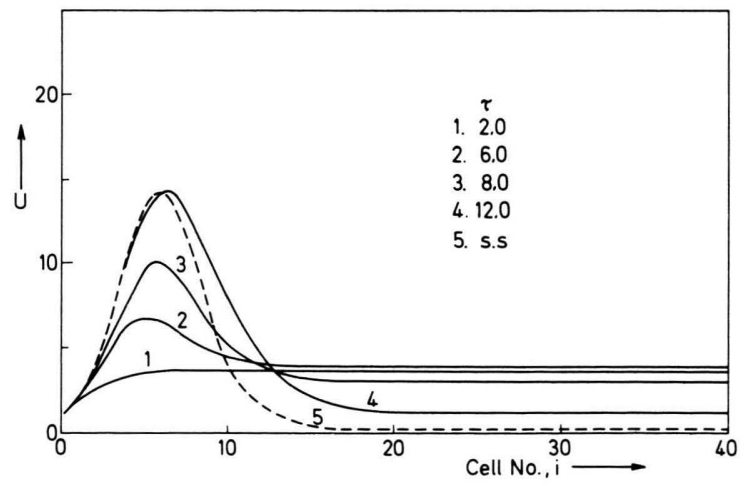

Fig. 9. Single standing wave in a tubular system. $\mathrm{Da}=0.50$, $\alpha_{1}=\alpha_{2}=\alpha_{3}=0.2$. 


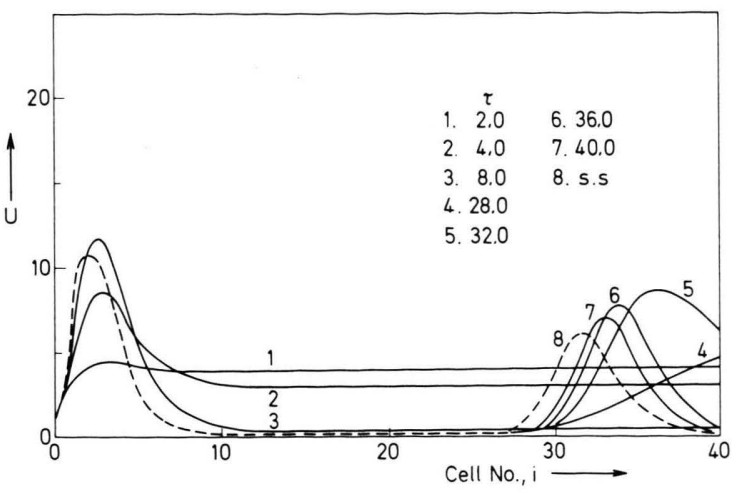

Fig. 10. Multiple standing waves in a tubular system. $\mathrm{Da}=1.00, \alpha_{1}=\alpha_{2}=\alpha_{3}=0.2$.

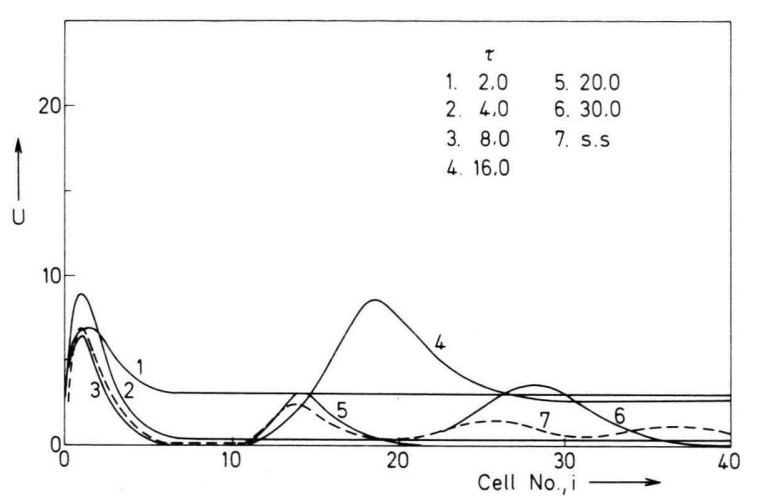

Fig. 11. Multiple standing waves in a tubular system. $\mathrm{Da}=2.00 \alpha_{1}=\alpha_{2}=\alpha_{3}=0.2$.
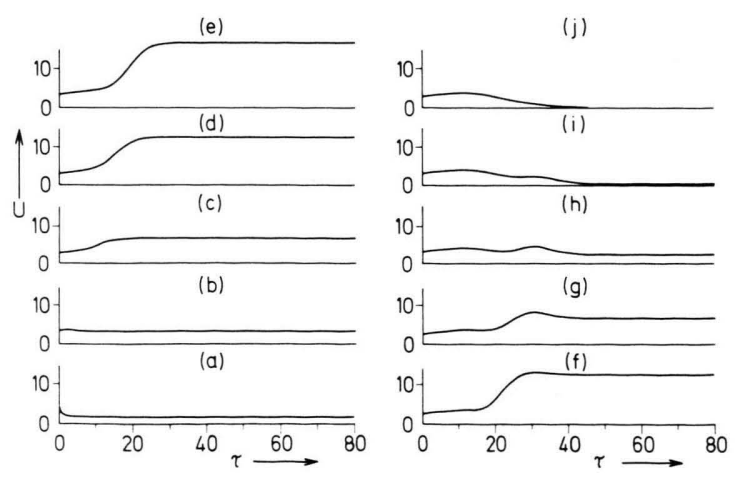

Fig. 12. Temporal behavior at different positions in a tubular system. $\mathrm{Da}=0.20, \alpha_{1}=\alpha_{2}=\alpha_{3}=0.2$. Cell No. is: (a) 1 , (b) 5 , (c) 9 , (d) 13 , (e) 17 , (f) 21, (g) 25 , (h) 29 , (i) 33, (j) 37 .
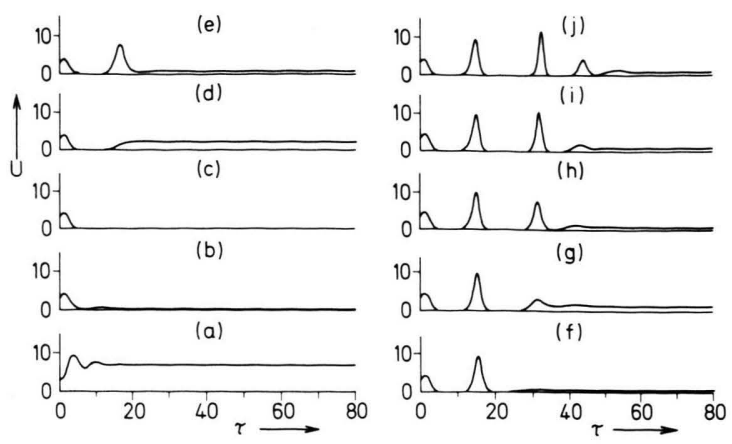

Fig. 13. Temporal behavior at different positions in a tubular system. $\mathrm{Da}=2.0, \alpha_{1}=\alpha_{2}=\alpha_{3}=0.2$. Cell No. is: (a) 1 , (b) 5 , (c) 9 , (d) 13 , (e) 17 , (f) 21 , (g) 25, (h) 29, (i) 33 , (j) 37 .

phenomenon may be elucidated in terms of a retriggering process or echo wave, originally proposed by Krinsky [25] for two coupled monostable generators. Consider two coupled elements at rest. Then, if one element is excited, the second also becomes excited after some delay, $\Delta$. If the delay time, $\Delta$, happens to lie between the refractory and the excitement time (i.e., $T_{\mathrm{R}}<\Delta<T_{\mathrm{E}}$ ), a retriggering wave may propagate in the distributed system. The time history for travelling and standing waves at different positions in the flow system is shown in Figs. 12 and 13, respectively. Figure 13 reminds one of the behavior of a retriggering wave. The dynamical behavior of the cell model thus turns out to be in a good qualitative agreement with that in the corresponding dispersion model. In the range of low Da's leading to a stable steady state, the backflow for mass has no qualitative effect on the dynamics of an autocatalytic reaction.

Based on the analysis for a single CSTR (and Fig. 2 as well) we can expect complex oscillatory behavior for high values of $\mathrm{Da}$. The temporal behavior of concentrations at different positions in the tubular system, calculated from the 1-D cell model with no backflow, $K_{m}=0.0$, is shown in Figure 14. The oscillatory profile of limit cycle type, generated in the first cell, propagates downstream the system. The amplitude of the "limit cycle" shrinks and the regular behavior changes toward complex multipeak and eventually chaotic-type oscillations. the transition to an irregular behavior occurs near cell twenty-one, see Figure 14f. A 3-dimensional plot clearly demonstrates the chang- 

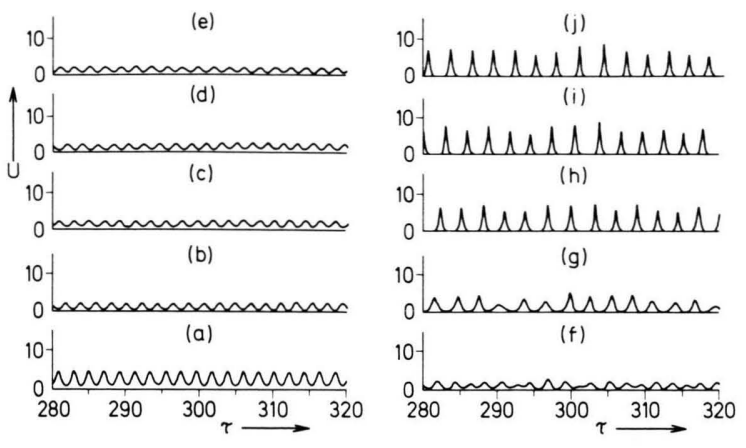

Fig. 14. Comparison of the oscillations at different positions in the 1-D cell model. $\mathrm{Da}=8.0, \alpha_{1}=\alpha_{2}=\alpha_{3}=0.2$. $K_{m}=0.0$. (a) 1 , (b) 5 , (c) 9 , (d) 13 , (e) 17 , (f) 21 , (g) 25 , (h) 29 , (i) 33 , (j) 37.

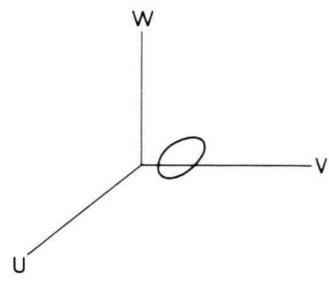

(a)

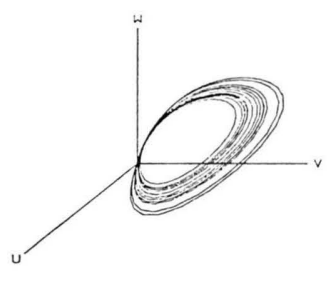

(c)

Fig. 15. Three-dimensional stereoplot at different positions in the 1-D cell model. $\mathrm{Da}=8.0, \alpha_{1}=\alpha_{2}=\alpha_{3}=0.2 . K_{m}=0.0$. Cell No. is: (a) 1, (b) 9 , (c) 33 .

ing type of the oscillations (see Figure 15a-15c). Chaotic-type behavior in the rear part of the system is depicted in Figure $15 \mathrm{c}$. This figure shows a "spiral-type" of chaotic oscillations. The corresponding spatial concentration profiles in the 1-D cell model at different times are shown in Figure 16.

The dynamics of the 1-D cell model can be viewed in terms of a set of forced oscillators. The output concentration from the preceding cell becomes the forcing function of the concentration
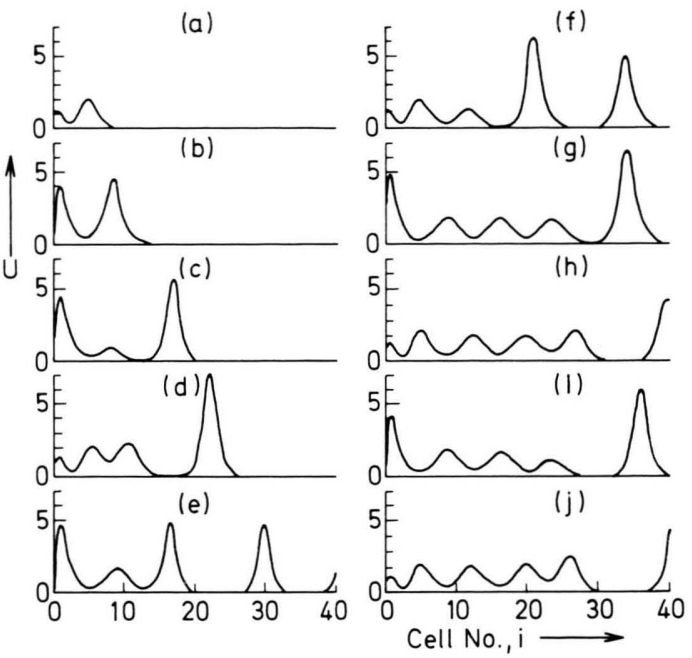

Fig. 16. Spatial concentration profiles in the 1-D cell model. $\mathrm{Da}=8.0, \alpha_{1}=\alpha_{2}=\alpha_{3}=0.2 . \quad K_{m}=0.0$. Time is: (a) 5.6, (b) 6.6, (c) 11.0, (d) 12.0, (e) 21.4 , (f) 22.4 , (g) 31.8 , (h) 32.8 , (i) 318.4 , (j) 328.4

feed to the next oscillator. When any of these oscillators is subjected to an oscillatory input, synchronization phenomena ca be expected. When the frequency of the input is fairly close to the natural frequency, a simple type of forced oscillation occurs. The range implied by "fairly close" depends on the amplitude of the input. The numerical study by Tomita and Kai [26] indicated that at input frequencies near twice the natural frequency, and for sufficiently large input amplitude, the subharmonic oscillation becomes unstable and may give rise to irregular behavior. Fujisaka [27], following [15a], also showed that a set of two or three coupled oscillators in a discrete model may exhibit chaotic behavior for a certain interval of parameters. Marek and Schreiber [28] also numerically observed chaotic behavior for two identical oscillators of a Brusselator model with different diffusion coupling for the two components. Figure 14 may be said to indicate subharmonic temporal and spatial bifurcations in a tubular system. In a 1-D cell model, the backflow has no significant qualitative effect on the dynamics of an oscillatory wave propagating in the reactor. However, an increasing backflow does give rise to a phase lag between the oscillations. This lag increases with time. The backflow rate for mass, $K_{m}$, is calculated by using the formula proposed in [29]. 

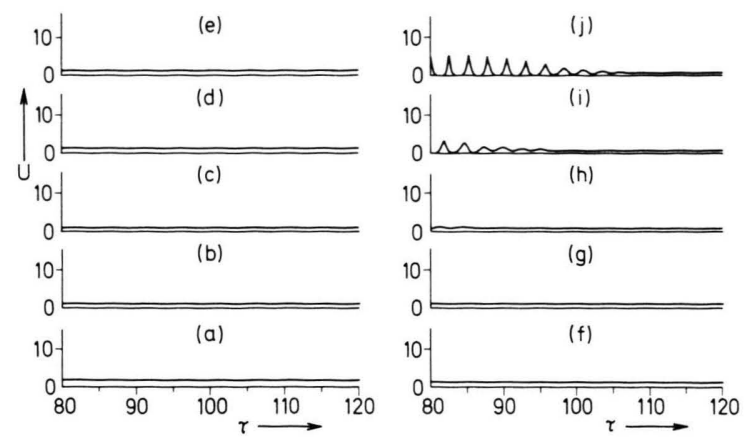

Fig. 17. Temporal behavior at different positions in the 1-D dispersion model. $\mathrm{Da}=8.0, \alpha_{1}=\alpha_{2}=\alpha_{3}=0.2$. $\mathrm{Pe}_{x}=$ $2.09(\mathrm{Re}=100)$. Axial coordinate is: (a) 1.0, (b) 5.0, (c) 9.0 , (d) 13.0 , (e) 17.0 , (f) 21.0 , (g) 25.0, (h) 29.0, (i) 33.0 , (j) 37.0 .
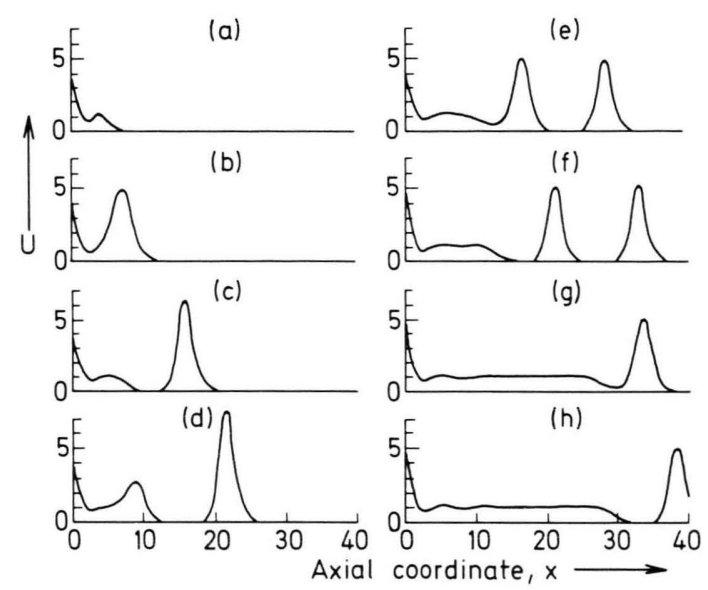

Fig. 18. Spatial concentration profiles in the 1-D dispersion model. $\mathrm{Da}=8.0, \alpha_{1}=\alpha_{2}=\alpha_{3}=0.2$. $\mathrm{Pe}_{x}=2.09(\mathrm{Re}=$ 100). Time is: (a) 5.6, (b) 6.6 , (c) 11.0 , (d) 12.0 , (e) 21.4, (f) $22.4,(\mathrm{~g}) 71.6,(\mathrm{~h}) 72.6$.

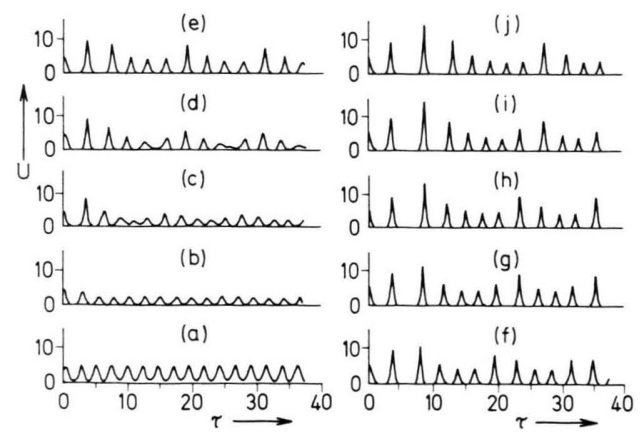

Fig. 19. Temporal behavior at different positions in the 1-D dispersion model. $\mathrm{Da}=8.0, \alpha_{1}=\alpha_{2}=\alpha_{3}=0.2$. $\mathrm{Pe}_{x}=$ $0.72(\mathrm{Re}=0.3)$. Axial coordinate is: (a) 1.0, (b) 5.0, (c) 9.0, (d) 13.0 , (e) 17.0 , (f) 21.0 , (g) 25.0 , (h) 29.0 , (i) 33.0 , (j) 37.0 .
The dynamic behavior of the 1-D dispersion model is shown in Figure 17. The corresponding cell model was displayed in Figure 14. For low values of the particle-related Peclet number, the temporal behavior of the dispersion model closely resembles, in a qualitative way, that observed in the cell model (cf. Fig. 19 with Figure 14).

An increasing value of $\mathrm{Pe}$ in the dispersion model suppresses the occurrence of spatial structures. This conclusion is in accordance with previous results for exothermic reactions [30]. A comparison of axial concentration profiles for the cell and the dispersion model show different types of behavior, see Figs. 16 and 18. We observed irregular dynamic behavior for the cell model while regular oscillations were always established for the disperion model.

\section{(2) Two-dimensional Systems}

Travelling, standing and oscillatory waves in the two-dimensional Deans-Lapidus cell model are shown in Figures 20-23. The oscillatory regular and irregular behavior in the axial direction reveals similarities to those observed for the one-dimensional cell model. The goal of the investigation of the 2-D model was to discover possibilities of symmetry-breaking of the lateral dimension in flow systems. However, no significant differences in the second space dimension have been observed for the systems under investigation, see Figure 21-23. Figure 23 shows the space distribution of concentration for the parameters corresponding to Figure 20. We see that the system does not show any tendency to create gradients in the radial direction. However, the autocatalytic flow system can be very sensitive in that respect. To prove this we introduced the $C_{1}$ component into the feed of only one cell $(i=0$, $j=1$ ). the symmetry-breaking response of the system is obvious from Figure 24. In this case, the radial diffusion downstream cannot eliminate the lateral perturbation, and extreme radial profiles result.

\section{Conclusions and Summary}

The dynamical behavior of autocatalytic reactions with a product inhibition term was studied both in distributed and lumped parameter flow systems. 


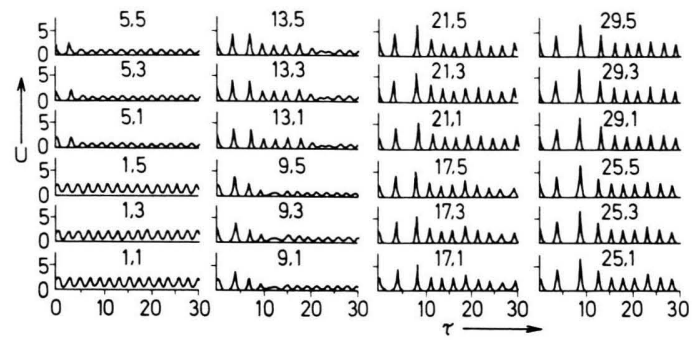

Fig. 20. Temporal behavior at different positions in a 2-D cell model. Da $=8.0, \alpha_{1}=\alpha_{2}=\alpha_{3}=0.2 . K_{m}=0.0, K_{r}=0.2$. The first and second numbers denote the cell number in axial and radial direction, respectively.

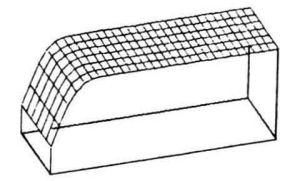

(a)

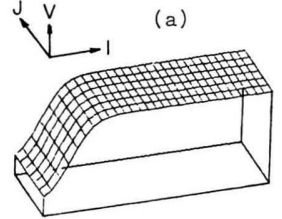

(b)

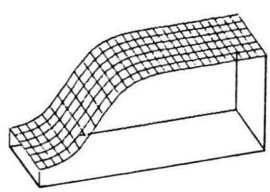

(c)

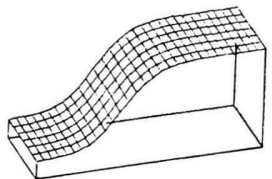

(d)

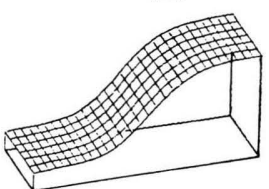

(e)

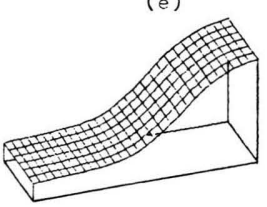

(f)
Fig. 21. Travelling waves in a 2-D cell modell. $\mathrm{Da}=0.02$, $\alpha_{1}=\alpha_{2}=\alpha_{3}=0.2, K_{m}=0.0, K_{r}=0.2$. Time is: (a) 2.0 , (b) 4.0 , (c) 8.0 , (d) 12.0 , (e) 16.0 , (f) 20.0 .

In a continuous stirred tank reactor, unicity of steady states exists. A linear stability analysis predicts a stable node, a stable focus, and a saddlefocus to be possible in the CSTR. Sustained oscillations around the unstable focus can occur for high values of the Damköhler number. The period of the oscillations increases with increasing flow rate.

In the distributed system, several kinds of wave phenomena such as travelling, standing and complex oscillatory waves were established.

For a low value of $\mathrm{Da}, \mathrm{Da} \cong 0.01-0.05$, travelling pseudo-constant pattern waves were detected. The characteristic steady state shows pattern formation in the spatial dimension.

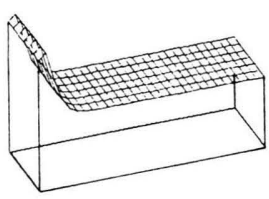

(a)

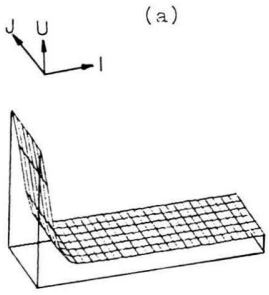

(b)

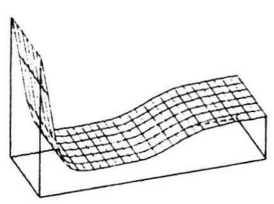

(c)

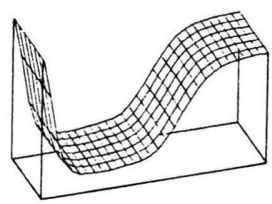

(d)

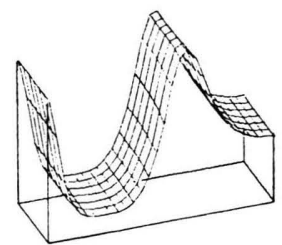

(e)

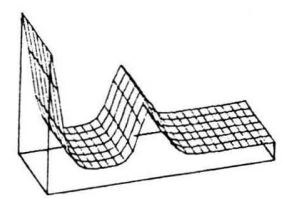

(f)

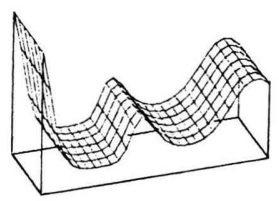

$(\xi)$

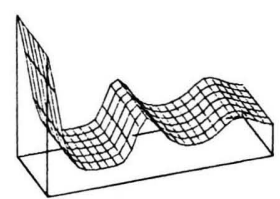

(h)
Fig. 22. Multiple standing waves in a 2-D cell model. $\mathrm{Da}=2.0, \alpha_{1}=\alpha_{2}=\alpha_{3}=0.2 . K_{m}=0.0, K_{r}=0.2$. Time is: (a) 2.0 , (b) 8.0 , (c) 13.0, (d) 14.0 , (e) 16.0 , (f) 20.0, (g) 30.0 , (h) s.s.

For intermediate values of $\mathrm{Da}, \mathrm{Da} \cong 0.1-2.0$, single or multiple standing waves were obtained. The number of standing waves increases with increasing values of $\mathrm{Da}$. A numerical simulation starting from different initial conditions revealed that unique multiple standing waves occur. The temporal behavior, at each position in the system, may exhibit the characteristics of a retriggering or echo wave, particularly so at $\mathrm{Da}=2.0$.

For high values of $\mathrm{Da}$, oscillating waves in the spatial dimension were observed. The results of simulation in a 1-D cell model show that regular oscillations can occur near the inlet but these oscillations change into irregular ones at a certain 


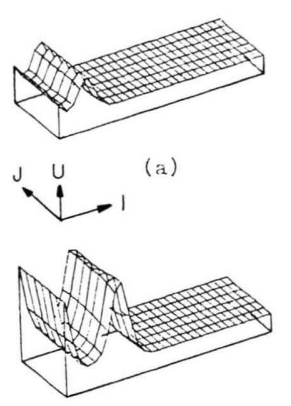

(b)

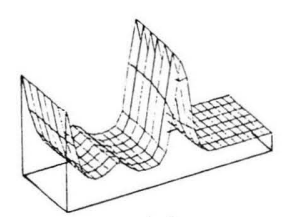

(c)

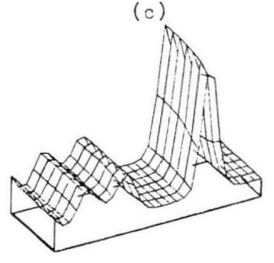

(d)
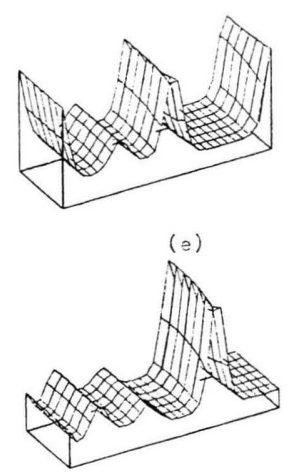

$(f)$

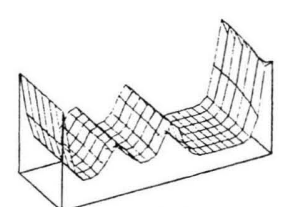

( g)

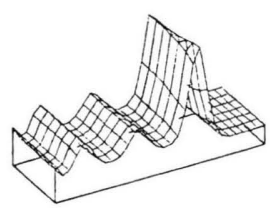

(n)

Fig. 23. Spatial concentration profiles in a 2-D cell model. $\mathrm{Da}=8.0, \alpha_{1}=\alpha_{2}=\alpha_{3}=0.2 . K_{m}=0.0, K_{r}=0.2$. Time is: (a) 5.6, (b) 6.6, (c) 11.0, (d) 12.0, (e) 21.4, (f) 22.4, (g) 31.8, (h) 32.8 .

axial position. This suggests that subharmonic limit cycle bifurcations, leading to chaotic behavior, are possible. The effect of backflow does not change the quality of the dynamical phenomena in the 1-D cell model except for the fact that increasing the backflow rate shifts the phase lag of the oscillation.

The dynamic properties of the 1-D dispersion model are strongly dependent on the value of the Peclet number. Higher values of Pe suppress oscilla-

[1] A. J. Lotka, Elements of Mathematical Biology, Dover 1956.

[2] G. Nicolis and I. Prigogine, Self-Organization in Non-equilibrium Systems, John Wiley, New York 1977.

[3] T. Erneux and M. Herschkowitz-Kaufman, Bull. Math. Biol. 41, 21 (1979).

[4] M. Herschkowitz-Kaufman, Bull. Math. Biol. 37, 589 (1975).

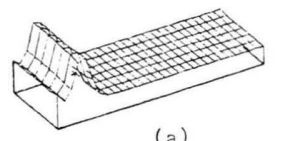

(a)

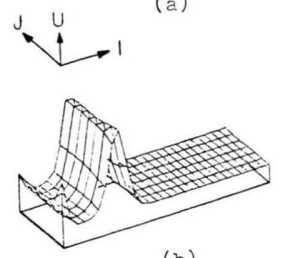

(b)
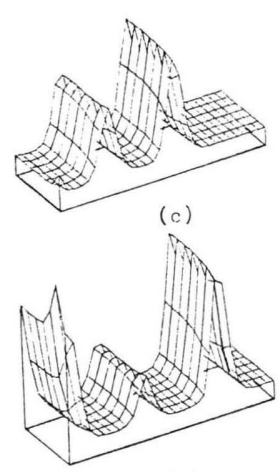

(d)

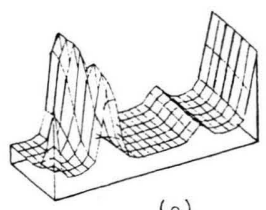

(e)

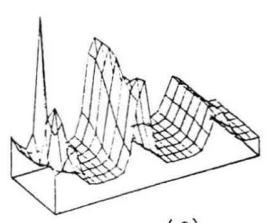

(f)

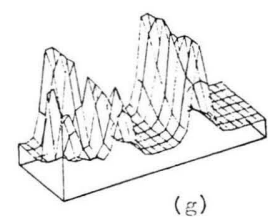

(g)

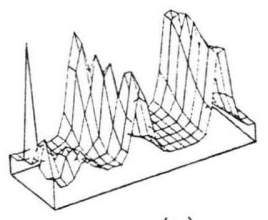

(h)
Fig. 24. Spatial concentration profiles in a 2-D cell model for inhomogeneous inlet concentrations (i.e. $U_{0, j}=0.0$ except $j=1) . \quad \mathrm{Da}=8.0, \quad \alpha_{1}=\alpha_{2}=\alpha_{3}=0.2 . \quad K_{m}=0.0$, $K_{r}=0.2$. Time is: (a) 5.6, (b) 6.6, (c) 11.0, (d) 12.0, (e) 21.4 , (f) 22.4 , (g) 31.8 , (h) 32.8 .

tions. The spatial profiles show a train of pulsating waves in the 1-D cell model and a single pulsating or solitary wave in the corresponding dispersion description.

In the 2-D model, also travelling and standing waves, analogous to those in the 1-D description, were observed. Permanent perturbation of one inlet cell induces a drastic change of the concentration profiles and may give rise to fingering waves.

[5] I. Prigogine and R. Lefever, J. Chem. Phys. 48, 1695 (1968).

[6] V. A. Vavilin and A. M. Zhabotinskii, Kinetics and Catalysis 10, 538 (1969).

[7] A. M. Zhabotinskii and A. N. Zaikin, Kinetics and Catalysis 12,516 (1971).

[8] D. A. Frank-Kamanetskii, Diffusion and Heat Transfer in Chemical Kinetics, Plenum Press, New York 1969. 
[9] I. Yamazaki, S. Nakamura, and K. Yokota, Nature, London 222, 794 (1969).

[10] D. F. Tatterson and J. L. Hudson, Chemical Eng. Commun. 1, 3 (1973).

[11] A. M. Zhabotinskii and A. N. Zaikin, J. Theor. Biol. 40, 45 (1973).

[12] M. Marek and Svobodová, Biophys. Chem. 3, 263 (1975)

[13] K. R. Graziani, J. L. Hudson, and R. A. Schmitz, Chem. Eng. J. 12, 9 (1976).

[14] R. A. Schmitz, K. R. Graziani, and J. L. Hudson, J. Chem. Phys. 67, 3040 (1977).

[15] O. E. Rössler, Nature London 271, 89 (1978).

[15a] O. E. Rössler, Z. Naturforsch. 31 a, 1168 (1976).

[16] R. H. Simoyi, J. C. Roux, and H. L. Swinney, Physica 8D, 257 (1983).

[17] J. C. Roux, Physica 7D, 57 (1983).

[18] V. Hlavacek, J. Sinkule, and M. Kubicek, J. Theor. Biol. 36, 283 (1972).

[19] J. Sinkule, V. Hlavacek, J. Vortruba, and I. Tvrdik, Chem. Engng. Sci. 29, 689 (1974).
[20] J. Puszynski, D. Snita, V. Hlavacek, and H. Hofmann, Chem. Engng. Sci. 36, 1605 (1981).

[21] H. A. Deans and L. Lapidus, AIChE J. 6, 656 (1960).

[22] P. Gray and S. K. Scott, Chem. Engng. Sci. 38, 29 (1983).

[23] L. M. Pismen, Chem. Engng. Sci. 38, 1950 (1980).

[24] J. C. Neu, SIAM J. Appl. Math. 36, 509 (1979).

[25] V. I. Krinsky, Science Publ. 20, 59 (1968) (in Russian).

[26] K. Tomita and T. Kai, Prog. Theor. Phys. 61, 54 (1979).

[27] H. Fujisaka, Phys. Lett. A 66, 450 (1978).

[28] M. Marek and I. Schreiber, Physica 5 D, 258 (1982).

[29] S. H. Kim and V. Hlavacek, On the Dynamics of Parabolic Equations Describing Diffusion, Convection and a Chemical Reaction, Physica $10 \mathrm{D}$ (in print).

[30] C. Y. Wen and L. T. Fan, Models for Flow Systems and Chemical Reactors, Marcel Dekker, Inc., New York 1975. 